\title{
Implementation of Biocatalysis in Continuous Flow for the Synthesis of Small Cyclic Amines
}

\author{
Eimear Hegarty and Francesca Paradisi*
}

\begin{abstract}
Significant progress has been made in establishing transaminases as robust biocatalysts for the green and scalable synthesis of a diverse range of chiral amines ${ }^{[1]}$ However, very few examples on the amination of small cyclic ketones have been reported. ${ }^{[2]}$ Cyclic ketones are particularly challenging for transaminase enzymes because they do not display the well-defined small and large substituent areas that are characteristic for the biocatalytic mechanism. In this work, we exploited the broad substrate scope of the (S)-selective transaminase from Halomonas elongata (HeWT) to develop an efficient biocatalytic system in continuous flow to generate a range of small cyclic amines which feature very often in pharmaceuticals and agrochemicals. ${ }^{[3]}$ Tetrahydrofuran-3-one and other challenging prochiral ketones were rapidly (5-45 $\mathrm{min}$ ) transformed to their corresponding amines with excellent molar conversion (94-99\%) and moderate to excellent ee.
\end{abstract}

Keywords: Biocatalysis · Continuous flow synthesis $\cdot$ Immobilisation · Small cyclic amines

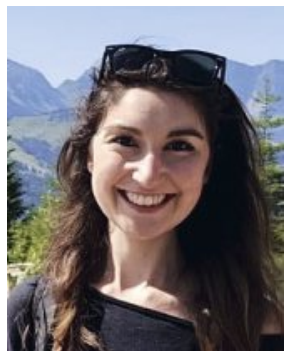

Eimear Hegarty graduated from the University of Limerick with a BSc in Industrial Biochemistry. In 2016, she joined Prof. Francesca Paradisi's research group at the University of Nottingham, where she obtained her $\mathrm{PhD}$ with a thesis on the development of novel biocatalytic routes for the synthesis of high-value chiral molecules. In 2019, she relocated with the group to the University of Bern as a postdoc. Her current research efforts are focussed on the implementation of biocatalytic processes in continuous flow.

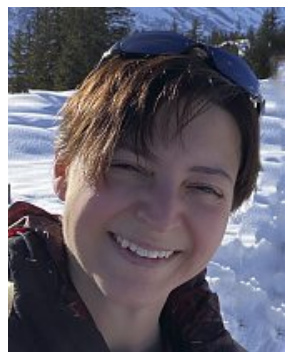

Prof. Francesca Paradisi is the Chair of Pharmaceutical and Bioorganic Chemistry at the University of Bern since 2019. She holds an MSc and a PhD in Chemistry from the University of Bologna and moved towards biocatalysis during her post doc at University College Dublin with Prof. Engel. She became an academic in 2006 at UCD and moved to the University of Nottingham, UK, in 2016. Biocatalysis as a sustainable approach to synthesis of valuable products is the focus of her research group. In particular, the group developed a number of enzyme-based processes in continuous flow, reducing the gap between academic discovery and industrial application.

\section{Introduction}

The asymmetric reduction of prochiral ketones with the formation of enantiomerically pure molecules is a fundamentally important reaction in synthetic organic chemistry and medicinal chemistry. These architectures find application in a wide range of fields, as well as serving as key intermediates in the synthesis of several pharmaceutical compounds. For example, the enantiopure chiral alcohol derived from tetrahydrofuran-3-one is used for the synthesis of the HIV inhibitor amprenavir and its prodrug form fosamprenaivr, while the amine analogue forms an integral structural feature in selective high affinity adenosine A1 receptor agonists, which have received considerable attention for their antiarrhythmic activity. ${ }^{[4,5]}$ Chiral manmade transition metal catalysts and certain organocatalysts have been applied successfully in ketone reductions. ${ }^{[6,7]}$ However, in all of these approaches, high enantioselectivty can be achieved only when the $\alpha$ and $\alpha^{\prime}$-substituents flanking the carbonyl functionality differ sterically. Spatially symmetric ketones, such as tetrahydrofuran-3-one and related substrates are particularly challenging in this regard, with asymmetric reductions typically resulting in poor enantioselectivities irrespective of the catalyst. To overcome these challenges, Pfizer developed a five-step process to obtain the chiral alcohol $(R)$-tetrahydrothiophene-3-ol which is a key component in the potent antibiotic sulopenem. ${ }^{[8]}$ While the desired alcohol is generally obtained in good yields and high optical purity (96-98\% ee) using this approach, the synthesis involves hazardous conditions (diazotization in step 1$)$, a sensitive reagent $\left(\mathrm{BH}_{3}\right.$-dimethylsulfide in step 2), high energy intermediates (epoxides in steps 3 and 4) and a noxious reagent ( $\mathrm{Na}_{2} \mathrm{~S}$ in step 5) (Scheme 1).

The uptake of biocatalysis as a useful tool for asymmetric synthesis has led to the development of a number of complemen-

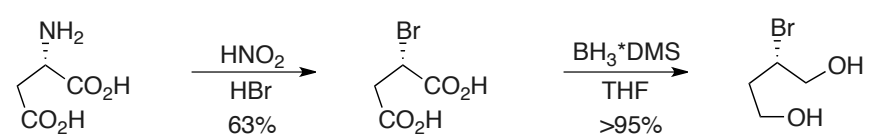

L-aspartic acid

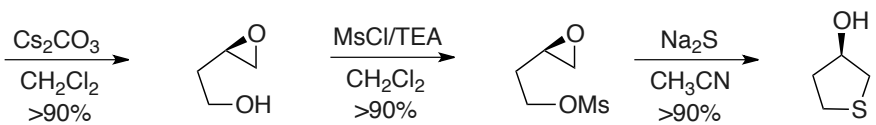

Scheme 1. Synthesis of $(R)$-tetrahydrothiophene-3-ol starting from L-aspartic acid. ${ }^{[8]}$ 
tary technologies that have enabled the intensification of industrially relevant biocatalytic processes. In particular, the transfer of enzyme-based processes into continuous flow is currently the focus of growing attention. ${ }^{[9]}$ While flow biocatalysis can be performed using immobilised whole-cells or cell-free enzymes, some excellent examples of cell-free reactions in continuous flow have appeared in the literature in recent years. ${ }^{[10-12]}$ For example, our group were the first to report on the use of $\omega$-transaminase $(\omega-\mathrm{TA})$ in continuous flow for the synthesis of a panel of aromatic amines. ${ }^{[13]}$ The enzyme, which was covalently immobilised onto a commercially available metal-derivatised epoxy resin proved to be highly stable in a packed bed reactor (PBR) at high flow rates. An in-line purification strategy was used, in which basification of the reaction stream and extraction with ethyl acetate, afforded the desired amine product. Traces of the unreacted aldehyde substrate in the organic phase were effectively trapped using a benzylamine functionalised resin. In addition excellent conversions and reaction times were achieved compared to those obtained in batch. Bearing this information in mind, we report herein on an enzyme-based system for the synthesis of a series of small cyclic molecules that are inherently difficult to produce using traditional chemical methods. Furthermore, the application of flow biocatalysis in this case effectively demonstrates how biocatalytic processes can easily be scaled-up, allowing for the direct, one-pot conversion of inexpensive and readily available compounds to high-value molecules, while also maintaining a very low environmental impact.

\section{Results and Discussion}

\section{Substrate Screening of Structurally Different $\omega$-TAs}

We started our studies on the sustainable formation of small cyclic amines by selecting two structurally different $\omega$-TAs from our toolbox of enzymes, a dimeric $\omega$-TA from Halomonas elongata HeWT and a tetrameric $\omega$-TA from Pseudomonas fluorescens PfTA. ${ }^{[14,15]}$ The previously reported screening for $H e \mathrm{WT}$ with this set of substrates ${ }^{[14]}$ is given in Table 1 for comparison. 1a was first employed on a $10 \mathrm{mM}$ scale using 1 equivalent of $(S)-\omega$ methylbenzylamine, $(S)$-MBA, as the amine donor. Remarkably, complete conversion to the corresponding amine $\mathbf{1 b}$ was achieved when $H e$ WT was employed as the biocatalyst, whereas PfTA reached a final conversion of $\sim 48.6 \%$, possibly due to thermodynamic limitations. Initial efforts to shift the equilibrium towards product formation, by increasing the concentration of the amine donor to $20 \mathrm{mM}$ were successful, resulting in a final conversion of $66.5 \%$ (Supplementary Information Table 3). However, further improvements could not be achieved as precipitation of the biocatalyst was observed at higher concentrations of $(S)$-MBA (concentrations $>20 \mathrm{mM}$ ). In terms of stereoselectivity, $(S)-\mathbf{1 b}$ was preferred in both cases, however the enantiomeric excess was low for both TAs (HeWT, 70\% (S); PfTA, 42\% (S)). Given the widespread occurrence of amino-substituted tetrahydrothiophenes, pyrrolidines and piperidines in pharmaceutically active compounds, the substrate scope of $\mathrm{HeWT}$ and PfTA was further probed with compounds $\mathbf{2 a - 4 a .} .^{[16,17]}$ Using the same reaction conditions as outlined above, $\mathrm{HeWT}$ was able to transform the selected compounds into the corresponding amines $(\mathbf{2 b}-\mathbf{4 b})$ with excellent molar conversions. PfTA, on the other hand, afforded moderate conversions in the case of compounds $\mathbf{2} \mathbf{a}$ and $\mathbf{4 a}$, while no conversion at all was detected in the case of $\mathbf{3 a}$ (Table 1). Optimisation of the reaction conditions again proved challenging due to the instability of the biocatalyst in the presence of high concentrations of $(S)$-MBA. Regarding the stereoselectivity, compound $\mathbf{2 b}$ was initially assumed to be $S$, in the absence of standards, by comparison with the other substrates. ${ }^{[14]}$ This has been recently re-evaluated by synthetic preparation of standards, to be, in fact, $R \cdot{ }^{[18]}$ The same enantiomer was produced by HeWT and
PfTA, however the ee was again low in both cases, at $66 \%$ and $20 \%$ respectively. Interestingly, the use of compounds with a methyl group at the 4-position (3a, 4a) gave significantly higher ee values, which suggests that the methyl group may help orientate the molecule in the active site in such a way that the formation of the $(S)$-enantiomer is favoured. To further probe this hypothesis, the crystal structure of $\mathrm{HeWT}$ (PDB: 6GWI) was used to dock selected compounds (1a and $\mathbf{4 a}$ ) from our panel into its active site. ${ }^{[14]}$

\section{Docking Analysis of Ketones 1a and 4a in the Active Site of HeWT}

$\mathrm{HeWT}$ is a homodimer, with an active site positioned at the interface of the monomers and a substrate binding region that is composed of a small and a large binding pocket. This characteristic architecture typically limits the substrate scope of wild-type $\omega$-TAs to ketones bearing at least one small substituent, not exceeding that of a methyl group. Our molecular docking studies have confirmed that small cyclic ketones are particularly challenging for $\mathrm{HeWT}$ as they evidently do not display the well-defined small and large substituents that are typically a prerequisite for the transaminase biocatalytic mechanism. ${ }^{[19]}$ Fig. 1(left) shows the docking of tetrahydrofuran-3-one (1a) in the active site of $\mathrm{HeWT}$. In this case, the tetrahydrofuranyl ring oxygen appears to form a hydrogen bond with the side chain of W56. This interaction anchors the substrate in the active site such that the reductive amination of the carbonyl is favoured. In the case of 1-methyl-3-piperidone (4a) the docking displaying the most favourable score places the substrate in a position that is further away from the PLP cofactor (3.23 $\AA$, Fig. 1(right)) when compared to $\mathbf{1 a}(2.70 \AA$, Fig. $1($ left)). Interestingly, the substrate is positioned in the vicinity of the large binding pocket. The high stereoselectivity achieved by $\mathrm{HeWT}$ in the case of $\mathbf{4 b}$, suggests that this orientation and binding mode favours the formation of the $(S)$-enantiomer.

\section{Continuous Flow Synthesis of Small Cyclic Amines}

The promising results obtained with $\mathrm{HeWT}$ in batch mode led us to explore the possibility of carrying out continuous reactions as an alternative strategy for the scale-up synthesis of the target amines. Compared to batch processes, performing reactions in continuous flow mode offer several benefits including better parameter control, reduced reaction times, improved yields and recycling of unreacted reagents. ${ }^{[20]}$ Specifically with respect to biocatalytic transformations, continuous flow can also offer additional benefits such as reduced enzyme inhibition through continuous product removal and facile downstream processing. ${ }^{[21]} \mathrm{HeWT}$ was therefore covalently immobilized onto a commercially available epoxy support as previously reported.[13] With an enzyme loading of $5 \mathrm{mg} / \mathrm{g}$ resin, the resulting biocatalyst displayed an activity of $38 \mathrm{U} / \mathrm{g}$ resin. A continuous flow reactor, packed with immobilized-HeWT, was subsequently set up and optimized for the preparation of $\mathbf{1 b}$. The substrate loading in this case was increased to 50 $\mathrm{mM}$, while the amine donor concentration was kept constant at 1 equivalent. Remarkably, complete molar conversion was achieved with a residence time of just 5 min. Furthermore, a downstream purification step to remove the acetophenone by-product, followed by liquid-liquid extraction facilitated the isolation of the final product with excellent yield $(91 \%)$. While full conversion of the starting material 1a was observed, the transamination reaction did not take place selectively. In fact, a significant loss in ee was observed with respect to the batch reactions (Table 2). A similar phenomenon was also observed in the case of $\mathbf{2} \mathbf{b}$. Although high conversion of $\mathbf{2 a}$ was achieved after 10 mins of residence time, the $e e$ of the final product was $26 \%(S)$. Interestingly, these results are much more comparable to those obtained in batch mode when the tetrameric TA from Pseudomonas fluorescence was employed as the biocatalyst. While the active sites of PfTA and HeWT were 
<smiles>O=C1CCCCC1</smiles><smiles>CC(C)C</smiles><smiles>NC1CCCCC1</smiles>

(S)-MBA
ACP

$\mathrm{HeWT}{ }^{[14]}$
Table 1. Transaminase mediated conversion of small cyclic prochiral ketones (1a-4a) in batch mode
$2 \mathbf{a}$

$1 \mathbf{a}$

Ketone

m.c. $[\%]$

$>99$<smiles>O=C1CCOC1</smiles><smiles>O=C1CCSC1</smiles>

$3 \mathbf{a}$

$4 a$<smiles>CN1CCC(=O)C1</smiles>

94

98

$>99$

$60(R)^{[18]}$

57.9

$20(R)$

$90(S)$

5.8

n.d

$90(S)$
PfTA

m.c. $[\%] \quad$ ee $[\%]$

48.6

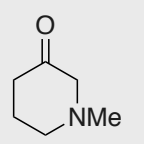

Molar conversions (m.c.), and enantiomeric excess (ee) for transaminase mediated conversion of tetrahydrofuran-3-one (1a), tetrahydrothiophene-3-one (2a), 1-methylpyrrolidin-3-one (3a) and 1-methyl-piperidin-3-one (4a). Final conversions were determined after $24 \mathrm{~h}$ of reaction. Biotransformations were performed with $10 \mathrm{mM}$ ketones, 1 equiv. (S)-(-)- $\alpha$-methylbenzylamine, $0.1 \mathrm{mM} \mathrm{PLP}, 1 \mathrm{mg} / \mathrm{mL}$ enzyme in $50 \mathrm{mM}$ phosphate buffer $\mathrm{pH} 8$ at $37^{\circ} \mathrm{C}$ (see Experimental details in Supplementary Information) $10 \%(\mathrm{~V} / \mathrm{V})$ DMSO was used in the case of 2a to improve solubility.
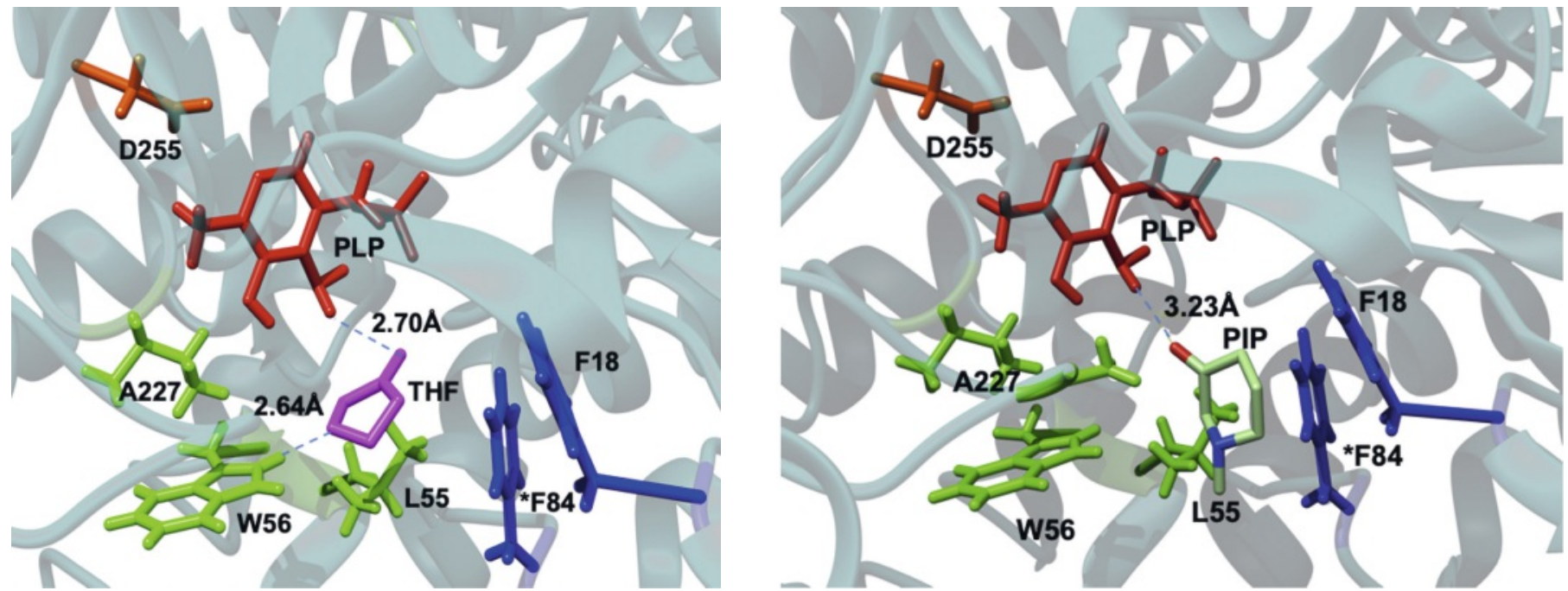

Fig. 1. Docking poses for tetrahydrofuran-3-one (left) and 1-methyl-3-piperidinone (right) in the active site of HeWT (PDB: 6GWI). Figures were generated using Chimera.

found to be very similar, the rigidity imposed by the additional subunits make it more challenging for PfTA to effectively differentiate between the $\alpha$ and $\alpha$-moieties flanking the carbonyl functional group of compounds $\mathbf{1 a}$ and $\mathbf{2 a}$. Similarly, the rigidification imposed upon immobilisation of $\mathrm{HeWT}$ is therefore thought to be the main reason for this unusual loss in $e e$.
Further reaction optimisation was required in the case of compounds 3a and 4a. High substrate loadings $(50 \mathrm{mM})$ led to poor molar conversions which may be attributed to substrate inhibition. As a compromise, the flow reactions were performed on a $25 \mathrm{mM}$ scale using 1 equivalent of $(S)$-MBA as the amine donor. Longer contact time with the biocatalyst (30-45 min) was required in 
Table 2. Continuous flow synthesis of small cyclic amines (1 $\mathbf{b}-\mathbf{4 b} \mathbf{b})$.

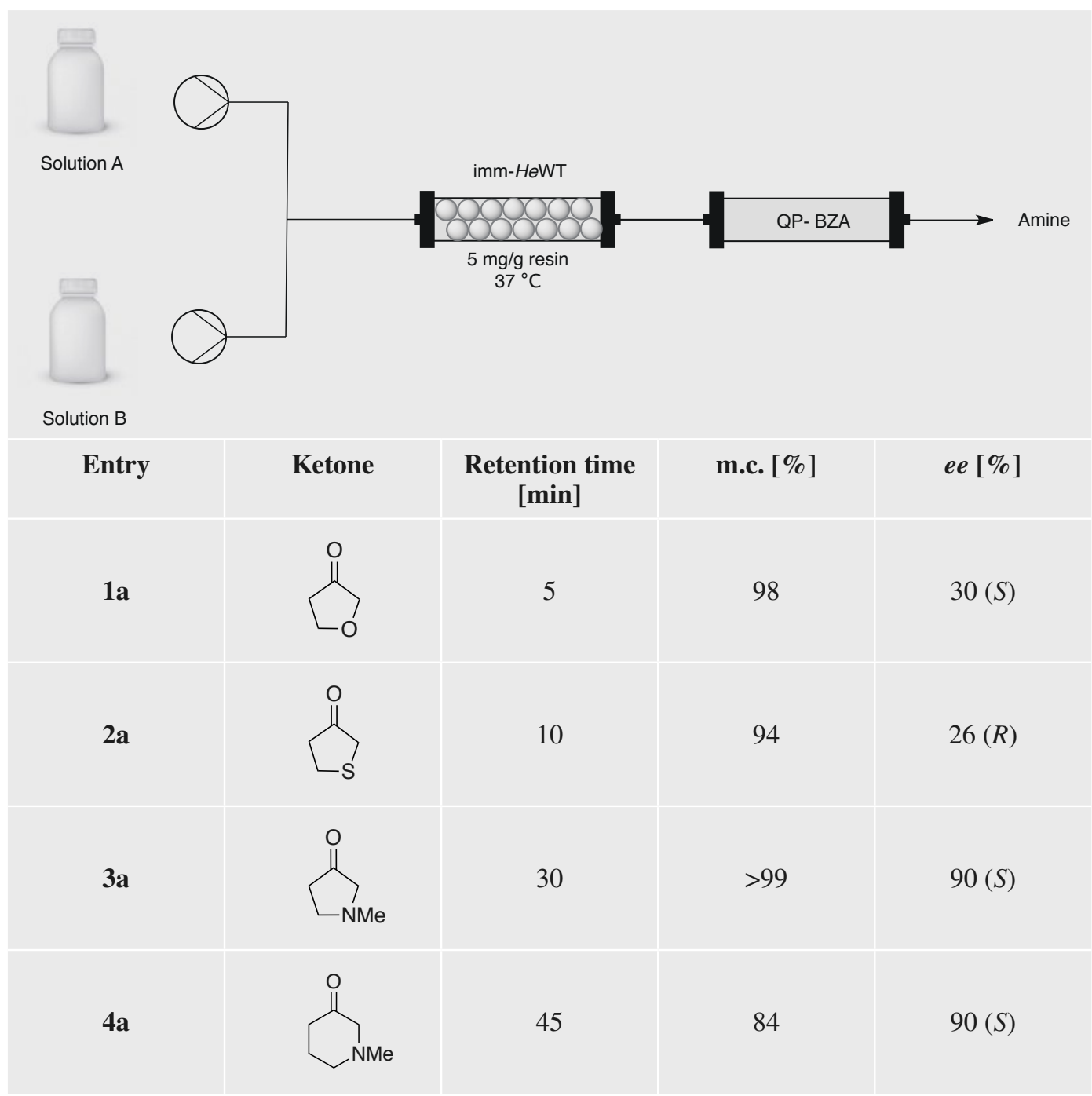

The reactions were performed in the presence of 25-50 mM ketone substrate (Solution A), 1 equiv. (S)-MBA and $0.1 \mathrm{mM}$ PLP (Solution B) in phosphate buffer $(50 \mathrm{mM}, \mathrm{pH} 8.0) .10 \%(\mathrm{v} / \mathrm{v})$ DMSO was used in the case of $2 \mathrm{a}$ to improve solubility. each case to reach high conversions. Furthermore, rigidification of the biocatalyst upon immobilisation had little effect on the enantioselectivity in these two cases. Both amine products were formed with excellent enantioselectivity (Table 2), consistent with those obtained in batch mode with the free enzyme.

\section{Conclusions}

In summary, we have developed a novel route for the preparation of a series of small cyclic amines using the $\omega$-TA from Halomonas elongata. Our system showcases how enabling technologies such as enzyme immobilisation and continuous flow can successfully be applied to develop robust biocatalytic strategies for the synthesis of molecules that are inherently difficult to access using traditional synthetic methodologies. While HeWT was found to be the preferred biocatalyst for the reductive amination of the target substrates, capable of achieving high molar conversions with exceptional reaction times in continuous flow, there are still a number of limitations that need to be addressed in order to develop a truly efficient biocatalytic process for the assembly of small cyclic amines. To meet this constraint, a deeper study of the active site of $\mathrm{HeWT}$ together with subsequent engineering efforts such as TCSM would be required to fine tune the stereoselectivity of the enzyme. ${ }^{[4,22]}$ In addition to this, alternative immobilisation techniques such as the fusion of a sacrificial protein to the target biocatalyst to facilitate site-specific covalent conjugation to the support may be useful in minimising rigidification and retaining catalytic activity upon immobilisation. ${ }^{[23]}$ Nevertheless, this work paves the way towards more sustainable and cost-effective manufacturing processes for an array of high value molecules.

\section{Supplementary Information}

Supplementary information is available on https://www.ingentaconnect.com/content/scs/chimia

Received: August 4, 2020

[1] I. Slabu, J. L. Galman, C. Iglesias, N. J. Weise, R. C. Lloyd, N. J. Turner, Catal. Today 2018, 36, 96, https://doi.org/10.1016/j.cattod.2017.01.025.

[2] E. Siirola, F. G. Mutti, B. Grischek, S. F. Hoefler, W. M. F. Fabian, G. Grogan, W. Kroutil, Adv. Synth. Catal. 2013, 355, 1703, https://doi.org/10.1002/ adsc. 201201057.

[3] L. Cerioli, M. Planchestainer, J. Cassidy, D. Tessaro, F. Paradisi, J. Mol. Catal. B Enzym. 2015, 120, 141, https://doi.org/10.1016/j.molcatb.2015.07.009.

[4] Z. Sun, R. Lonsdale, A. Ilie, G. Li, J. Zhou, M. T. Reetz, ACS Catal. 2016, 6, 1598, https://doi.org/10.1021/acscatal.5b02752.

[5] E. Elzein, R. Kalla, X. Li, T. Perry, T. Marquart, M. Micklatcher, Y. Li, Y. Wu, D. Zeng, J. Zablocki, Bioorganic Med. Chem. Lett. 2007, 17, 161, https://doi.org/10.1016/j.bmcl.2006.09.065.

[6] R. Noyori, Angew. Chem. Int. Ed. 2002, 41, 2008, https://doi. org/10.1002/1521-3773(20020617)41:12<2008::AID-ANIE2008>3.0. $\mathrm{CO} ; 2-4$. 
[7] E. J. Corey, C. J. Helal, Angew. Chem. Int. Ed. 1998, 37, 1986. [8] Liang, E. Mundorff, R. Voladri, S. Jennet, L. Gilson, A. Conway, A. Krebber, J. Wong, G. Huisman, S. Truesdell, J. Lalonde, Org. Process Res. Dev. 2010, 14, 188, https://doi.org/10.1021/op9002714.

[9] L. Tamborini, P. Fernandes, F. Paradisi, F. Molinari, Trends Biotechnol. 2018, 36, 73, https://doi.org/10.1016/j.tibtech.2017.09.005.

[10] M. L. Contente, F. Paradisi, Nat. Catal. 2018, 1, 452, https://doi.org/10.1038/ s41929-018-0082-9.

[11] C. J. Hartley, C. C. Williams, J. A. Scoble, Q. I. Churches, A. North, N. G. French, T. Nebl, G. Coia, A. C. Warden, G. Simpson, A. R. Frazer, C. N. Jensen, N. J. Turner, C. Scott, Nat. Catal. 2019, 2, 1006, https://doi. org/10.1038/s41929-019-0353-0.

[12] D. Roura Padrosa, A. I. Benítez-Mateos, L. Calvey, Green Chem. 2020, 22 , 531, https://doi.org/10.1039/D0GC01817A.

[13] M. Planchestainer, M. L. Contente, J. Cassidy, F. Molinari, L. Tamborini, F Paradisi, Green Chem. 2017, 19, 372, https://doi.org/10.1039/c6gc01780k.

[14] M. Planchestainer, E. Hegarty, C. M. Heckmann, L. J. Gourlay, F. Paradisi, Chem. Sci. 2019, 10, 5952, https://doi.org/10.1039/c8sc05712e.

[15] D. Roura Padrosa, R. Alaux, P. Smith, I. Dreveny, F. López-Gallego, F. Paradisi, Front. Bioeng. Biotechnol. 2019, https://doi.org/10.3389/fbioe.2019.00282

[16] N. Kim, K. M. Meyers, J. L. Mendez-Andino, N. C. Warshakoon, W. Ji, J. A. Wos, A. Colson, M. C. Mitchell, J. R. Davis, B. B. Pinney, O. Reizes, X. E. Hu, Med. Chem. Lett. 2006, 16, 5445, https://doi.org/10.1016/j. bmcl.2006.07.053.
[17] H. Sun, D. O. Scott, ACS Med. Chem. Lett. 2011, 2, 638, https://doi. org $/ 10.1021 / \mathrm{ml} 200117 \mathrm{z}$.

[18] C. M. Heckmann, L. J. Gourlay, B. Dominguez, F. Paradisi, Front. Bioeng. Biotechnol. 2020, 8, 707, https://doi.org/10.3389/fbioe.2020.00707.

[19] L. Leipold, D. Dobrijevic, J. W. E. Jeffries, M. Bawn, T. S. Moody, J. M. Ward, H. C. Hailes, Green Chem. 2019, 21, 75, https://doi.org/10.1039/c8gc02986e.

[20] M. L. Contente, S. Farris, L. Tamborini, F. Molinari, F. Paradisi, Green Chem. 2019, 21, 3263, https://doi.org/10.1039/c9gc01374a.

[21] J. Britton, S. Majumdar, G. A. Weiss, Chem. Soc. Rev. 2018, 47, 5891, https://doi.org/10.1039/c7cs00906b.

[22] Z. Sun, R. Lonsdale, L. Wu, G. Li, A. Li, J. Wang, J. Zhou, M. T. Reetz, ACS Catal. 2016, 6, 1590, https://doi.org/10.1021/acscatal.5b02751.

[23] M. Planchestainer, D. R. Padrosa, M. L. Contente, F. Paradisi, Catalysts 2018, 8, https://doi.org/10.3390/catal8010040.

\section{License and Terms}

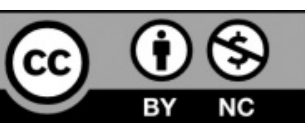

This is an Open Access article under the terms of the Creative Commons Attribution License CC BY_NC 4.0. The material may not be used for commercial purposes.

The license is subject to the CHIMIA terms and conditions: (http:// chimia.ch/component/sppagebuilder/?view=page \&id=12).

The definitive version of this article is the electronic one that can be found at https://doi.org/10.2533/chimia.2020.890 